\title{
Psychiatric Pharmacogenomics: How to Integrate into Clinical Practice
}

\author{
Stephen M. Stahl \\ ISSUE: \\ Pharmacogenomic testing can be integrated into modern mental health practices \\ to help select psychotropic drugs for individuals who have failed first-line \\ evidence-based treatments. This can be done by the process of "equipoise"- \\ namely, balancing the weight of all available evidence. That evidence now includes \\ not only diagnosis-specific treatment guidelines and "personalized" patient \\ information, such as an individual's specific symptom profile, past response to \\ medications, side effects, family history, and patient preference, but also "precision \\ medicine," which incorporates the ever-expanding base of pharmacogenomic \\ evidence for how an individual's own biomarkers alter the odds for that individual's \\ treatment response or treatment intolerance.
}

\section{Take-Home Points}

- Suboptimal treatment responses are common in psychopharmacology

- Most psychopharmacologic treatment guidelines emphasize first-line therapy selection, and not what to do when several options are not effective or are not tolerated.

- Best practice for drug selection for treatment-resistant patients in the absence of randomized controlled trials is shifting from trial-and-error approaches informed by "personalized" clinical data from a specific individual to incorporating as well the results of "precision medicine" using an individual's own biomarkers.

- Pharmacogenomic "precision" testing can increasingly inform drug selection in treatment-resistant patients and currently includes state-of-the-art pharmacokinetic and pharmacodynamic genomic markers, with epigenetic and other biomarkers poised to enter clinical practice as wel.

Dr. Stahl was awarded the 2016 David A. Mrazek Award in Pharmacogenomics by the American Psychiatric Association, and this article is based on the David A. Mrazek Award Lecture, which Dr. Stahl delivered at the American Psychiatric Association meeting in Atlanta, Georgia, May 2016.

\section{Introduction}

Psychiatric pharmacogenomics and biomarkers are on the cutting edge between research and clinical practice, where the action can be quite turbulent and even controversial. ${ }^{1-13}$ Payors who do not want to reimburse useful if expensive tests are accused of being greedy or Luddites; laboratories are accused of profit mongering with tests that do not alter clinical practice or patient outcomes; clinicians are accused of over-interpreting test results. So, what is the truth? Should we just stick with the classical model of mental health practice (Table 1)? Maybe the truth is actually a bit of all the above. Here we will take a quick inventory of the state of the art of "precision medicine" for mental health practices.

\section{Genomics to Diagnose?}

Currently, it is not at all clear what role genomics and biomarkers have in the diagnosis of mental illnesses, or in risk for mental illnesses. ${ }^{4,11,13}$ Application of new tests for clinical diagnoses of mental illnesses is proving problematic, not least because diagnosis in mental health is undergoing a paradigm shift from categorical 


\section{BRAINSTORMS -Clinical Neuroscience Update}

\section{Table 1. Classical model of psychiatric practice}

- Use treatment guidelines/evidence-based medicine.

- What you do when there is no evidence:

- Use experience from training and practice.

- Use trial-and-error enhanced by personalized patient information, such as symptom profile, side effects, reactions to prior treatments, family history, and patient preference, as well as prescriber experience and preference.

- Use intuition.

- Potential pitfalls:

- Recency effect: If you have just seen something, you may think it occurs more frequently than it really does.

- Significant emotional event effect: If there is a dramatic effect, you may think this also occurs more frequently than it really does.

- Eminence-based medicine: Expert-based experience may be biased.

(DSM, Diagnostic and Statistical Manual) to dimensional (symptom domains that cut across numerous psychiatric disorders). ${ }^{14}$ We really do not have diseases in mental health practice, so much as diagnoses that are collections of diseases. Thus, it already seems clear there will not be a single test for any diagnosis, and that at best a portfolio of numerous biomarkers may become useful for potentially identifying many genetic or biological expressions of the same diagnosis. So, biomarkers for diagnosis do not appear to be ready now for "prime time" in clinical practice.

\section{Genomics for Initial Drug Selection?}

Much of the effort in the development of biomarkers has been dedicated to finding tests that would tell us what drug to prescribe or what drug not to prescribe, in order to find a drug with efficacy but not side effects for an individual patient. So, where are we in the process of integrating such "precision medicine" into drug selection in mental health practices? One thing is already clear from pharmacogenomics in psychiatry: Tests do not select drugs. Prescribers do.

That is, it is highly unlikely that any single test will ever dictate what drug to prescribe or not to prescribe in most cases. There is no known single gene for any major psychiatric disorder nor for any drug response to a psychiatric disorder, nor is one ever likely to be found, since genes do not code for psychiatric disorders, nor for psychiatric symptoms, nor for drug responses to psychiatric symptoms (Table 2) ${ }^{14}$ Instead, genes code for proteins
Table 2. Genes and responses to psychotropic drugs

- There is no known single gene for any major psychiatric disorder, nor for any drug response to a psychiatric disorder, nor is one ever likely to be found.

- Genes do not code for psychiatric disorders.

- Genes do not code for psychiatric symptoms.

- Genes do not code for drug response to psychiatric disorders.

- Genes code for proteins and epigenetic factors, many of which regulate the efficiency of information processing in brain circuits, which can be visualized with neuroimaging techniques.

- Psychiatric research is attempting to link treatment response to neuronal circuits upstream and to numerous regulatory genes downstream.

- Pharmacogenomic testing adds to the balance of the evidence of what to do, and the data behind each test is ever-evolving although the genetic test results themselves will never change.

and epigenetic factors that regulate the efficiency of information processing in brain circuits, and that can be increasingly visualized with neuroimaging techniques. ${ }^{14,15}$ Rather than looking for a single gene that regulates drug response, psychiatric research is instead currently attempting to link treatment response to a portfolio of genes that regulate brain circuits that are the substrates of various psychiatric symptoms (Table 2). ${ }^{14,15}$

Such a portfolio of biomarkers will hopefully show which drugs will be somewhat more likely to work or to cause a side effect in a given patient. Right now, however, it is not clear that the available genomic tests add substantial value proportionate to their cost for selection of first-line treatments of mental disorders. ${ }^{1-13}$ For selection of a first-line therapy, current treatment guidelines alone may be most cost effective. If there is a place for current pharmacogenomic testing, it may be in the selection of drugs for patients who are treatmentresistant or treatment-intolerant to trials of evidencebased therapies (Tables 3 and 4), particularly when these test results are augmented with therapeutic drug-level monitoring combined with classical approaches to selecting treatments (Table 1$)^{1-24}$

\section{Genomics for Drug Selection in Treatment Resistance/Intolerance?}

One of the most daunting tasks for a modern mental health professional is selecting treatment for a patient 


\section{BRAINSTORMS-Clinical Neuroscience Update}

Table 3. Pharmacogenomic testing in modern psychiatric

practice

- A strategy to use when there is no evidence from large, randomized, controlled trials or all these approaches have failed

- A dozen or two well-studied single nucleotide polymorphisms (SNPs) associated with drug response

- Some highly replicated, others not

- Each individual SNP has effects that are only weak/small in determining overall treatment response

- Most are neurobiologically plausible

- Danger of "over-interpretation" by patients or eager/ unsophisticated clinicians

- Orients the advanced prescriber for treatment-resistant cases toward recent data organized along a neurobiological perspective

- Useful in developing rational hypotheses for novel treatments or combinations in individual patients who are resistant to multiple agents

- Gives hope to patients, enhances optimism and motivation of prescribing clinicians, and provides a scientific basis (if weak and evolving) to selection of agents in the absence of controlled trials

who has failed to tolerate or respond adequately to numerous evidence-based treatments. Because large, randomized, controlled trials of these so-called treatment-resistant or treatment-intolerant patients are few, it leaves such patients and their prescribers to ponder, "What do you do when there is no evidence?"

As mentioned above, the answer in the past has been the classical model of best practices for such patients (Table 1). The question now is whether pharmacogenomic testing has advanced sufficiently such that the information obtained is worth its cost for the treatment-resistant patient. If so, this would mean combining the classical approach of empiric case-based evidence from use of drugs that have a pharmacologic rationale for an individual patient, including a specific patient's unique information (personal symptom profile, prior clinical response or nonresponse to other agents, particular side effects experienced, family history, and preferences), with information from genotyping (both pharmacokinetic and pharmacodynamic markers; phenotyping therapeutic drug levels; and obtaining results from various epigenetic, proteomic, and neuroimaging biomarkers as well). Such an approach seeks to employ the best of the art of psychopharmacology with the best of available science for mental health patients who are most in need of effective,
Table 4. Proposal for a modern model of psychiatric practice

for treatment resistance

- Exhaust evidence-based solutions.

- Think.

- Take another history, including from a new informant.

- Reconsider the diagnosis.

- eg, TRD may be bipolar, mixed features, pseudobulbar affect, dementia, etc.

- Collect new data, including therapeutic drug levels and available pharmacogenomic markers.

- Use this new information to rebalance the evidence (equipoise) and come up with a genetically informed, neurobiologically empowered, data-oriented, novel, and rational treatment or combination.

tolerable treatments. So far, the empiric evidence is that this approach might "work" for the treatment-resistant patient, especially therapeutic blood monitoring for treatment-resistant psychosis with violence in forensic settings, ${ }^{16,17}$ and pharmacokinetic/pharmacodynamic genomic markers for treatment-resistant depression. ${ }^{18-24}$ "Work" in this context means symptom improvement, better tolerance to medication, and cost savings.

\section{It's the Strategy, Not the Test Result}

So far, therefore, there have been some surprises from pharmacogenomic testing as it enters mental health practice (Table 3). First, as mentioned above, we now know that no single test will tell us what to prescribe or what not to prescribe for a given patient. It is clear that each test result only "biases" us a small amount for or against a given drug choice, and that information must be balanced (ie, with equipoise) with other personal information from that unique patient. Second, perhaps the most important outcome from pharmacogenomic testing is not necessarily the specific test result, but how this testing leads to drug selection that improves outcomes and reduces costs. ${ }^{18-24}$ That is, interpreting pharmacogenomic test results orients the advanced prescriber's thinking along a neurobiological perspective in order to select treatments that are biologically plausible, rather than just utilizing intuition, habit, or trial and error. This appears to have the potential to improve drug selection. ${ }^{18-24}$ Third, we now know that how one utilizes pharmacogenomic test results is not that different from how one utilizes any other personalized clinical information from a given patient. That is, each bit of information from a specific patient, whether 


\section{BRAINSTORMS -Clinical Neuroscience Update}

clinical or pharmacogenomic testing, contributes at best a very small amount to the variance explaining why someone responds or fails to respond, or tolerates or fails to tolerate a given drug or drug class. Having more information from pharmacogenomic test results provides additional individualized data for a given patient to help weigh the many factors in favor or against prescribing any given drug.

Some skeptics conclude from all of this that no biomarker or genomic test is valuable enough to be part of the standard of psychiatric care, nor useful enough to be reimbursed. Integrating new technologies into clinical practice has always been a messy affair as we learn whether clinical outcomes are better when the test results are utilized than when they are not. Early adopters strive to discover the best utility of new information, while nay-sayers and especially payors remain doubtful. Some practitioners may be more comfortable at the present time using the timehonored classical approach (Table 1). On the other hand, early initiators of new technology who study the literature and learn how to properly interpret evolving test results may prefer a more cutting-edge, if controversial, approach (Table 4). That approach is not to take a classical trial-and-error approach to selecting treatments, but instead to put the results of pharmacogenomic testing into the decision-making formula by pursuing a genetically informed, neurobiologically empowered, data-oriented, novel, and rational approach to selecting a treatment or combination that is already showing signs of yielding better symptomatic outcomes, better dosing, and reduced cost of treatment (Table 4). ${ }^{18-24}$

\section{References:}

1. Stahl SM. Personalized Medicine, pharmacogenomics and the practice of psychiatry: on the threshold of predict therapeutics in psychopharmacology? CNS Spectr. 2008; 13(2): 115-118.

2. Mrazek DA. Psychiatric Pharmacogenomics. New York: Oxford University Press; 2010.

3. Bousman CA. Commercial pharmacogenomics-based decision support tools in psychiatry. Lancet Psychiatry. 2016; 3(6): 585-590.

4. Braff DL, Freedman R. Clinically responsible genetic testing in neuropsychiatric patients: a bridge too far and too soon. $A m \mathrm{~J}$ Psychiatry. 2008; 165(8): 952-955.

5. Malhotra AK, Murphy GM, Kennedy JL. Pharmacogenetics of psychotropic drug response. Am J Psychiatry. 2004; 161(5): 780-796

6. Cross-Disorder Group of the Psychiatric Genomics Consortium Identification of risk loci with shared effects on five major psychiatric disorders: a genome wide analysis. Lancet. 2013, 381(9875): 1371-1379.
7. Lotrich FE. The emerging potential of pharmacogenetics in psychiatry. Am J Psychiatry. 2012; 169(7): 681-683.

8. Beevers CG, McGeary JE. Therapygenetics: moving towards personalized psychotherapy treatment. Trends Cogn Sci. 2012; 16(1): 11-12.

9. Falcone M, Smith RM, Chenoweth MG, et al. Neuroimaging in psychiatric pharmacogenetics research: the promise and the pitfalls. Neurospsychopharmacology. 2013; 38(12): 2327-2337.

10. Varela MA, Roberts TC, Wood MJA. Epigenetics and ncRNAs in Brain Function and Disease: mechanisms and prospects for therapy. Neurotherapeutics. 2013; 10(4): 621-631.

11. Perlis RH. Can single genes matter in a polygenic world? Biol Psychiatry. 2010; 68(9): 783-784.

12. Caspi A, Hariri AR, Holmes A, Uher R, Moffitt TE. Genetic sensitivity to the environment: the case of the serotonin transporter gene and its implications for studying complex diseases and traits. Am J Psychiatry. 2010; 167(5): 509-527.

13. Niculescu AB, Levey D, Le-Niculescu H, Niculescu E, Kurian SM, Salomon D. Psychiatric mood biomarkers: avoiding jumping to premature negative or positive conclusions. Mol Psychiatry. 2015; 20(3): 286-288.

14. Stahl SM. The last Diagnostic and Statistical Manual (DSM): replacing our symptom-based diagnoses with a brain circuit-based classification of mental illnesses. CNS Spectr. 2013; 18(2): 65-68.

15. Stahl SM. Stahl's Essential Psychopharmacology. 4th ed. New York: Cambridge University Press; 2014.

16. Morrissette DA, Stahl SM. Treating the violent patient with psychosis or impulsivity utilizing antipsychotic polypharmacy and high-dose monotherapy. CNS Spectr. 2014; 19(5): 439-448.

17. Stahl SM, Morrissette DA, Cummings M, et al. California State Hospital Violence Assessment and Treatment (Cal-VAT) guideline. CNS Spectr. 2014; 19(5): 449-465.

18. Ruaño G, Szarek BL, Villagra D, et al. Length of psychiatric hospitalization is correlated with CYP2D6 functional status in inpatients with major depressive disorder. Biomark Med. 2013; 7(3): 429-439.

19. Herbild L, Andersen SE, Werge T, Rasmussen HB, Jürgens G. Does pharmacogenetic testing for CYP450 2D6 and 2C19 among patients with diagnoses within the schizophrenic spectrum reduce treatment costs? Basic Clin Pharmacol Toxicol. 2013; 113(4): 266-272.

20. Brennan FX, Gardner KR, Lombard J, et al. A naturalistic study of the effectiveness of pharmacogenetic testing to guide treatment in psychiatric patients with mood and anxiety disorders. Prim Care Companion CNS Disord. 2015; 17(2). doi: 10.4088/PCC.14m01717.

21. Fagerness J, Fonesca E, Hess GP, et al. Pharmacogenetic-guided psychiatric intervention associated with increased adherence and cost savings. Am J Manag Care. 2014; 20(5): e146-e156.

22. Hall-Flavin DK, Winner JG, Allen JD, et al. Using a pharmacogenomic algorithm to guide the treatment of depression. Transl Psychiatry. 2012; 2: e172.

23. Hall-Flavin DK, Winner JG, Allen JD, et al. Utility of integrated pharmacogenomic testing to support the treatment of major depressive disorder in a psychiatric outpatient setting. Pharmacogenet Genomics. 2013; 23(10): 535-548.

24. Winner JG, Carhart JM, Altar CA, Allen JD, Dechairo BM. A prospective, randomized, double-blind study assessing the clinical impact of integrated pharmacogenomic testing for major depressive disorder. Discov Med. 2013; 16(89): 219-227. 